\title{
Perceptual clustering in auditory streaming
}

\author{
Nathanael Larigaldie \\ Psychology Department \\ Durham University, Durham, UK \\ Tim Yates \\ Department of Psychology \\ University of Birmingham \\ Ulrik R. Beierholm \\ Psychology Department \\ Durham University, Durham, UK \\ Corresponding author: ulrik.beierholm@durham.ac.uk
}

\section{Abstract}

\begin{abstract}
Perception relies on being able to segregate stimuli from different objects and causes to perform inference and further processing. For simple binary stimuli (e.g. auditory and visual) we have models of how the human brain can perform such causal inference, but the complexity of the models increases dramatically with more than 2 stimuli. To characterize human perception with more complex stimuli we have developed a Bayesian inference model that allows an unlimited number of stimulus sources to be considered: it is general enough to allow any discrete sequential cues, from any modality. The model uses a non-parametric prior, hence increased complexity of the signal does not necessitate more parameters. The model not only determines the most likely number of sources, but also specifies the source that each signal is associated with. As a test application we show that such a model can explain several phenomena in the auditory stream perception literature, that it gives an excellent fit to experimental data,
\end{abstract}


and that it makes novel predictions that we confirm experimentally. These results have implications not just for human auditory temporal perception but for a large range of other perceptual phenomena.

Keywords- perception, causal inference, occam's razor, gestalt psychology, non-parametric, bayesian inference

\section{Introduction}

Ambiguity in perceptual systems is a blight for inference. When we hear two sounds sequentially, we may infer that they came from two different sources, e.g. birds $A$ and $B$, or the same source repeated. A third sound is heard - are the generating sources $A A A, A A B, A B A, A B B$, or $A B C$ (see Fig. 1 for the possible generative models)? By the time four, five and six sounds are heard the number of possible combinations reaches 15,52 , and 858 . The ambiguity breeds to generate a combinatorial explosion, and yet the human auditory system is able to reliably allocate multiple sources of sound in complex, real world situations. Features of the signal are consistently associated with different sources, allowing us to keep track of a speaker's voice and the wail of an ambulance siren, separately from the noise of background traffic and falling rain.

Inferring the generative model that caused the observed stimuli is a general problem faced by the perceptual system. To perform such a task for simple stimuli the brain relies on causal inference [2, 3], probabilistically estimating the most likely cause of the stimuli in the environment. This has been shown to be a good model of perceptual inference for ambiguous stimuli, when they are in small numbers, i.e. two. However, the increase in the number of stimuli causes the complexity of possible generative structures to rapidly increase (Fig. 1), rendering a causal inference strategy that relies on enumerating all possible structures impossible.

An important realisation is however, that given a specific set of a large number of stimuli, this process is essentially one of clustering, combining together stimuli that are similar while keeping separate from those dissimilar. This idea, that perception involves clustering, has a long history in the Gestalt psychology literature, although not always expressed in those terms [4]. However the brain would need to choose the right number of clusters and have a way to specify the prior expectations over clusters, which is hard before even knowing the number of stimuli.

The key proposal in this paper is that the brain can perform this clustering process using a modern version of 'Occam's Razor', non-parametric Bayesian clustering. With this approach the numbers of clusters do not have to be pre-specified, instead the algorithm adapts the number of clusters to the data. Likewise, there is no need for a large number of parameters to specify the prior expectations, instead a single meta-parameter specifies the degree of clustering. Individual data points (stimuli) are assigned to different groups (or clusters) based on the other existing data points. This type of algorithm is renown for allowing the complexity of the model to grow with the data set $[5,6]$ as a larger number of clusters can emerge as the number of data points increases. 
a)

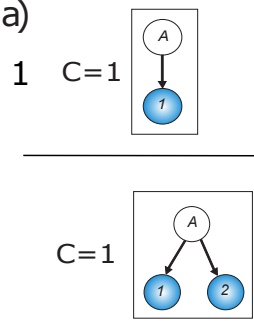

2

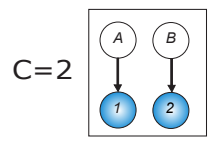

$C=1 \overbrace{1}^{A}$

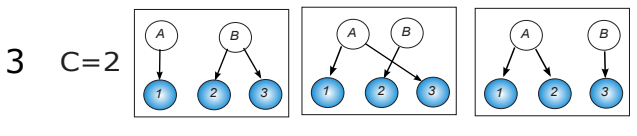

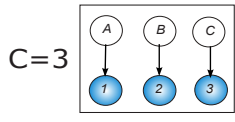

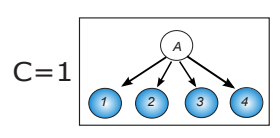

b)

1 stream

c)

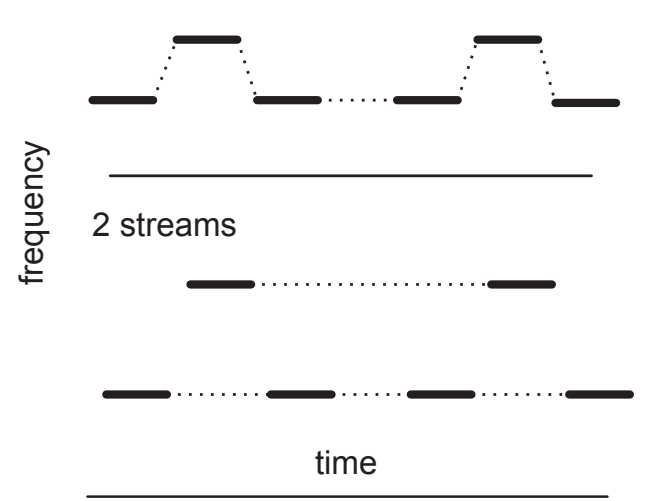

Any \# of streams

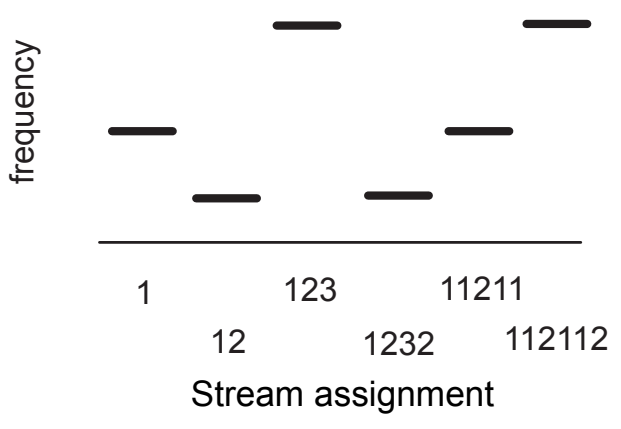

4

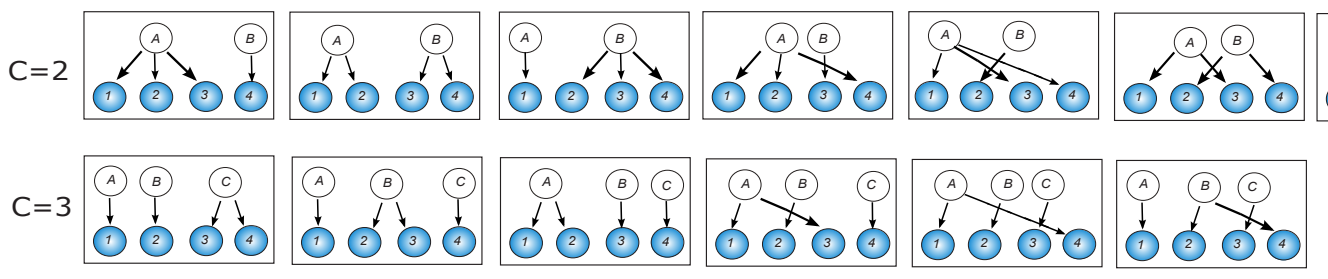

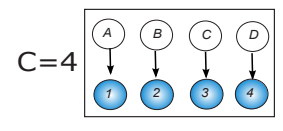

Figure 1. a) Graphical illustration of clustering problem in causal inference. As the number of stimuli increases $(1,2,3,4, \ldots)$ the number $(C)$ of potential causes increases at the same rate, while the number of combinations of causes that could have generated the stimuli increases according to the number of ways to partition a set of $n$ objects into $k$ non-empty subsets. It is easy to differentiate between the two potential generative structures when there are only two stimuli, but much harder when four stimuli can be created from fifteen different generative structures. b) Example of auditory tones being segregated into one or two streams, using 'galloping' stimuli similar to [1]. c) Example of a series of potential stimuli with a representative assignment of tones to streanos below. As each tone is presented the observer reassigns the entire set of tones to streams $(1->12->123$ etc.). The brain has to decide how to assign each tone into an unknown number of streams, a type of clustering problem. 
Non-parametric Bayesian inference has previously been used in cognitive and perceptual studies ([7, 8]), but not to study the segmentation of perceptual cues.

To exemplify how human perception of large number of sources can be modeled by non-parametric Bayesian inference, we will present modeling and experimental results on auditory stream segregation.

\section{Auditory stream segregation}

For several decades, the human ability to segregate sequential sounds into streams corresponding to sources has been investigated using simple sequences of either pure tones or more complex sounds (reviewed in [9]). The time interval between tones, their pitch difference and the duration of a sequence are among the factors that play an important role ([10]; [11]; [1]): explanations of how the factors are used based on principles such as Gestalt laws and Occam's razor have been incorporated into the conceptual model of Bregman ([12]).

Descriptive models based on peripheral excitation ([13]), coherence of coupled oscillators ([14]) and cortical streaming modules ([15]) provide mechanisms to estimate the number of streams, but do not specify which sound is associated with which source. While some of the models are expandable to allow more sources to be inferred, it is not known if they would cope with the combinatorial explosion. Furthermore, Moore and Gockel ( [16] ) conclude from an extensive review of the literature that any sufficiently salient factor can induce stream segregation. This indicates that a more general model of inference is needed, that can incorporate any auditory perceptual cue and multiple sounds with different sources.

If ambiguity is a blight for inference, regularities in natural signals are the cure. Not all combinations of signal sources are equally likely - when perceptual systems generate a model of the world, we assume that they infer the most likely interpretation because the perceptual systems are optimized to the statistics of natural signals ([17, 18]). Bayesian inference has had considerable success in modeling many visual and multi-sensory percepts as a generative, probabilistic process ([19-21]). Despite these successes, we still have no general, principled model of how the auditory system solves the source inference problem.

A Bayesian approach to auditory stream segregation (based on sequential sampling) has been used to model the dynamics of perceptual bistability ([22]) but assumes that only two percepts are possible. Turner ([23]) has developed methods of analyzing statistics of sounds based on Bayesian inference, and constructed a model to synthesize realistic auditory textures. While inference in the model can qualitatively replicate many known auditory grouping rules, the expected number of sources in the environment has to be specified. In a natural scenario an observer would have to infer the number of sources that are present in the environment due to the inherent uncertainty.

In our model the probability of many alternative stream configurations (given the input signal) are calculated and the percept generated corresponds to the most probable configuration. The probabilities are calculated using Bayes' 
rule to combine the likelihood of generating a signal given a postulated stream configuration, with the prior probability of sounds being associated with different sources. The likelihood and prior probability distributions are iteratively updated in a principled manner as information accumulates.

The forms of the distributions are presumably optimized to natural signal statistics: the likelihood distribution we use is based on consideration of the physical limitations of oscillators. However, the framework of the model allows formulations of multiple explanatory factors, such as those determined by Bregman ([12]) from psychophysics experiments, to be simply incorporated in the distributions. Furthermore, while the current study uses simple pure tones (replicating work by Bregman), the framework allows more complex cues from audition and other modalities to be used as long as their perceptual difference can be quantified.

\section{Model}

Pure tones are the indivisible atoms of input to the model - each being assigned to just one sound source, or stream. Inspired by the work done on non-parametric priors $([7,24,25])$ we assume the existence of an infinite number of potential sources, leading to a sequence of tones with pitch $f_{1}, f_{2} \ldots$, onset time $t_{1}^{o n}, t_{2}^{o n} \ldots$ and an offset time, $t_{1}^{o f f}, t_{2}^{o f f} \ldots$ and the sound sources/streams that generated the tones are denoted by positive integers $S_{1}, S_{2} \ldots$ We rename the sources when necessary so that the first tone heard will always be generated by source 1 (i.e. $S_{1}=1$ ), and a subsequent $i$ th tone can be associated with source $1: \max \left(S_{1} \ldots S_{i-1}\right)+1$.

\section{Generative model}

Given a source $S_{i}$ we assume that the frequency of tone $i$ is governed by physical constraints and statistical regularities of the source. If two sequential sounds with the frequencies $f_{i-1}$ and $f_{i}$ are produced by the same source, $S_{i}=S_{i-1}$, the pitch cannot change at an infinitely fast rate: to make an oscillator change its frequency discontinuously would require an infinite impulse of energy. We assume that, all things being equal, a pure tone sound source is most likely to continue oscillating at the same frequency as it has in the past, and the probability decreases with $\Delta f=f_{i}-f_{i-1}$ but increases with $\Delta t=\left(t_{i}^{o n}-t_{i-1}^{o f f}\right)$. More specifically we assume a normal probability distribution:

$$
p\left(f_{i}, t_{i}^{o n} \mid S_{i}=S_{i-1}, f_{i-1}, t_{i-1}^{o f f}\right)=\frac{1}{\sqrt{2 \pi \sigma^{2}}} \exp ^{-\frac{\left(\frac{\Delta f}{\Delta t}\right)^{2}}{2 \sigma^{2}}}
$$

where $\sigma$ is a constant. We here assume that the observer has a perfect noise free access to the generated auditory frequencies. 
For successive sources, we assume that sources that have been active previously are more likely to be active again, but do not provide a limit to the number of sources that $N$ tones can be generated from. Concretely we assign the probability of a source $j$ generating the $i$ th tone according to a Chinese Restaurant Process (CRP) [5], which can be considered as an extension of Occam's rule. If the number of tones previously assigned to source $j=S_{i}$ is given by $n_{i}=\sum_{k=1}^{i-1} \delta\left(S_{i}-S_{k}\right)$ then

$$
\begin{array}{r}
p\left(S_{i}=j \mid S_{1} \ldots S_{i-1}\right)=n_{i} /(i-1+\alpha) \\
\text { if } n_{i}>0 \\
p\left(S_{i}=j \mid S_{1} \ldots S_{i-1}\right)=\alpha /(i-1+\alpha) \\
\text { if } n_{i}=0
\end{array}
$$

and where $\delta$ is the discrete Kronecker delta function $(\delta(0)=1$, but 0 elsewhere). Intuitively, the more tones have already been assigned to a source, the more likely that tone $i$ will also be assigned to it ("rich-get-richer" scheme).

\section{Inference}

The task of the observer is to infer the sources generating each of the tones, i.e. to find the sequence $S_{1} S_{2} S_{3} \ldots$ that maximize $p\left(S_{1} S_{2} S_{3} \ldots \mid f_{1} f_{2} f_{3} \ldots, t_{1}^{o n} t_{2}^{o n} t_{3}^{o n} \ldots, t_{1}^{o f f} t_{2}^{o f f} t_{3}^{o f f} \ldots\right)$, as illustrated in figure 1 . For simplicity of writing we will refer to the properties of tone $i$ as $x_{i}$ in place of the set $\left(f_{i}, t_{i}^{o n}, t_{i}^{o f f}\right)$

As an example, we use a sequence of three tones $x_{1}, x_{2}, x_{3}$, for which the observer wishes to infer the likely sources $S_{1}, S_{2}, S_{3}$. Thus the probability $p\left(S_{1}, S_{2}, S_{3} \mid x_{1}, x_{2}, x_{3}\right)$ that a sequence of three tones was generated by sources $S_{1}, S_{2}, S_{3}$, has to be calculated over the five possible combinations: $\left[S_{1}=1, S_{2}=1, S_{3}=1\right]$, [ $S_{1}=1, S_{2}=1, S_{3}=$ 2], $\left[S_{1}=1, S_{2}=2, S_{3}=1\right],\left[S_{1}=1, S_{2}=2, S_{3}=2\right],\left[S_{1}=1, S_{2}=2, S_{3}=3\right]$ corresponding to the five unique configurations of sources generating three sounds. Note that the first source is always assigned the value 1 , the next different source is assigned 2, etc.

Bayes' rule relates each conditional probability (posterior distribution) to the likelihood $p\left(x_{1}, x_{2}, x_{3} \mid S_{1}, S_{2}, S_{3}\right)$ of each configuration of sound sources generating the sequence of tones, by

$$
p\left(S_{1}, S_{2}, S_{3} \mid x_{1}, x_{2}, x_{3}\right)=p\left(x_{1}, x_{2}, x_{3} \mid S_{1}, S_{2}, S_{3}\right) p\left(S_{1}, S_{2}, S_{3}\right) / Z
$$

where $Z$ is a normalization constant, and $p\left(S_{1} S_{2} S_{3}\right)$ is the prior probability of the particular configuration of sound sources, regardless of the frequency, etc. of the tones

Assuming conditional independence of the tones and tone-source causality, this can be rewritten as 


$$
\begin{aligned}
p\left(S 1, S_{2}, S_{3} \mid x_{1}, x_{2}, x_{3}\right) & =p\left(x_{3} \mid S_{1}, S_{2}, S_{3}\right) / p\left(x_{3}\right) \times p\left(S_{1}, S_{2}, S_{3} \mid x_{1} x_{2}\right) \\
& =p\left(x_{3} \mid S_{1}, S_{2}, S_{3}\right) / p\left(x_{3}\right) \times p\left(S_{3} \mid S_{1}, S_{2}\right) p\left(S_{1}, S_{2} \mid x_{1} x_{2}\right)
\end{aligned}
$$

The final term is the posterior generated from the first two tones. The latter two terms can be considered together as the prior for the third source, allowing us to use an iterative approach to the inference. After each tone we grow the tree of possible source sequence (e.g. $11 \rightarrow 111$ and 112), by multiplying the previous posterior $p\left(S_{1}, S_{2} \mid x_{1}, x_{2}\right)$ with two terms; the likelihood $p\left(x_{3} \mid S_{1}, S_{2}, S_{3}\right)$ and a prior for how likely the next 'branch' is, $p\left(S_{3} \mid S_{1}, S_{2}\right)$.

We now consider how to determine the likelihood and prior probabilities. The first source can only be associated with one source, so $p\left(S_{1}=1\right)=1$. The principle of Occam's razor would suggest that $p\left(S_{1}=1, S_{2}=1\right)>p\left(S_{1}=\right.$ $1, S_{2}=2$ ), i.e. if we haven't heard any of the sounds, the most probable acoustic scene is the simplest one: all sounds come from the same source. The value of $p\left(S_{1}=1, S_{2}=1\right)$ for an individual can be determined from fitting their data, and the value $p\left(S_{1}=1, S_{2}=2\right)$ is simply $1-p\left(S_{1}=1, S_{2}=1\right)$. The values may depend on factors such as the environment, which are not considered in the model: natural signal statistics may provide guidance for how the prior probabilities are assigned.

Regarding the likelihood function, for new tone $i$ the observer assumes the generative probability $p\left(x_{i} \mid S_{i}, x_{i-k}, S_{i}=\right.$ $S_{i-k}$ ), where tone $i-k$ was the latest tone inside stream $S_{i}$. Note that this applies even when the sounds generated by the same source are separated by one or more sounds associated with different sources (e.g., $\left(S_{1}=1, S_{2}=\right.$ $\left.2, S_{3}=1\right)$ ). The only transition that matters is that between the most recent tone and the last tone in the same stream, so if three tones $x_{1}, x_{2}$ and $x_{3}$ had all been associated with the same stream (e.g. $\left(S_{1}=1, S_{2}=1, S_{3}=1\right)$ ), we would only consider the transition from $x_{2}$ to $x_{3}$, whereas if $x_{2}$ was associated with a different stream (e.g. $\left(S_{1}=1, S_{2}=2, S_{3}=1\right)$ ), we would only consider the transition from $x_{1}$ to $x_{3}$.

If a sound comes from a new source, then we assume that the likelihood is independent of previous tones:

$$
p\left(f_{i} \mid S_{1} \neq S_{i}, S_{2} \neq S_{i}, \ldots S_{i-1} \neq S_{i}\right)=\frac{1}{\sqrt{2 \pi \sigma^{2}}} \exp ^{-\frac{\left(f_{i}-f_{0}\right)^{2}}{2 \sigma^{2}}}
$$

where $f_{0}$ is the midpoint of the range of auditory frequencies presented for the trial.

The final model has two parameters, $\alpha$ and $\sigma$, plus a parameter for the steepness of the response variability (given by softmax function) for each subject $\beta$. For details of implementation of the model see Methods. 


\section{Results}

To evaluate the performance of the model, we made qualitative comparisons with studies in the literature and quantitative comparison with experimental data. We furthermore tested a prediction from the model using a novel experimental paradigm.

\section{Modeling}

\section{Time}

A well-known basic stream segregation phenomena (e.g. [11]) shows that increasing the speed at which auditory tones increases the probability that tones are perceived as coming from separate streams. To examine this in the model we recreate the second experiment of Bregman and Campbell ([11]), showing in Fig 2 that while a sequence of six slowly presented tones is assigned a low posterior probability of originating from different sources (and should therefore be assigned the same stream), as the speed of presentation is increased the probability of originating from two sources increases drastically (implying subjects should segregate the streams).

\section{Galloping effect}

Several studies have shown how the effect of frequency and time can interact. Van Norden ([1]) found that in a repeating Low-High-Low sequence of tones the subjects would report one or two streams as a function of both the difference in auditory frequency and the speed of presentation (interstimulus interval). In our computational model (see Fig. 2) the likelihood term directly depends on both of these factors, and the prior probability again constrains the observer from segregating tones into more streams. We replicate the results of this study in Experiment 1 below.

\section{Cumulative}

The galloping sequence Low-High-Low has also been used to highlight the effect of the accumulation of information (Fig. 3). Bregman ([26]) showed that a short sequence of tones tends to lead to the percept of a single stream, whereas the accumulation of information causes the tones to segregate into two streams. For the model, this effect is due to the non-parametric prior initially assigning a low probability to the possibility of two streams, before more information is gathered. 
a)

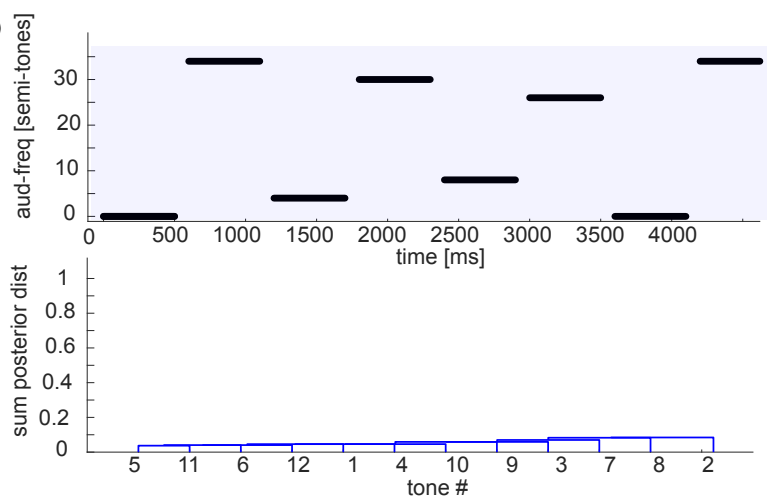

c)
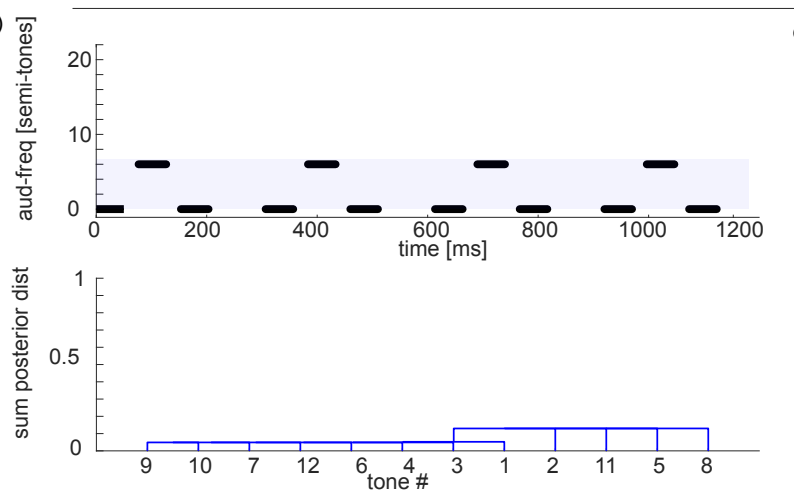

b)
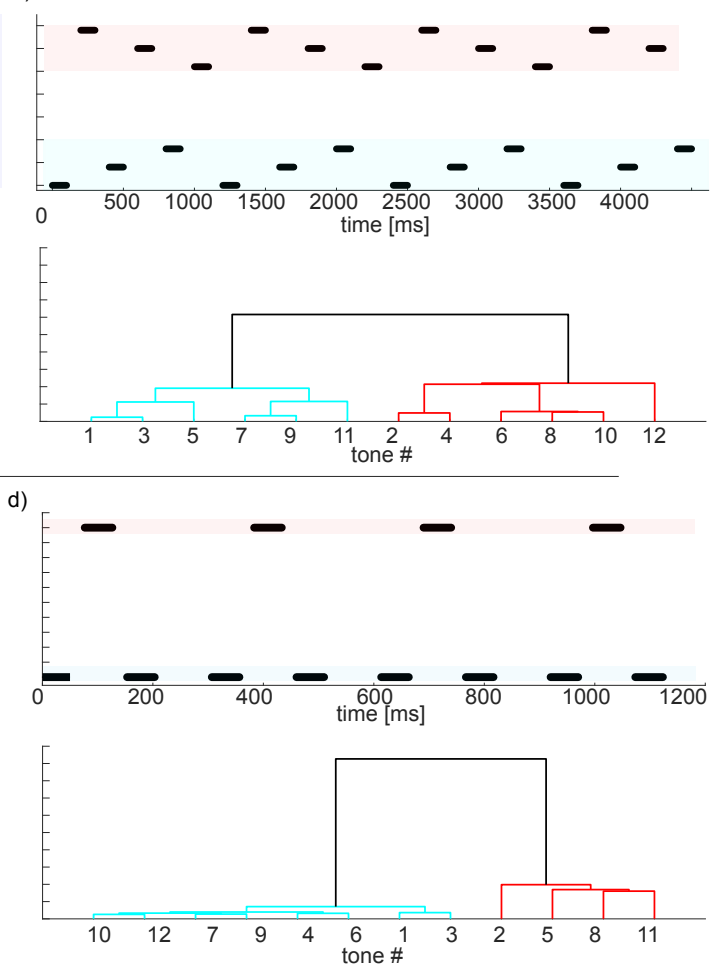

Figure 2. a-d) Stimuli used in experiments from [11] (second experiment), highlighting how the speed of presentation affects perception of streams of tones. Stimuli are shown at the top, bottom are dendrogram tree-plots based on the posterior distribution over clustering. A unique colour is assigned to clusters with more than 50 percent distance from other clusters. a) Slow sequence, ISI $100 \mathrm{~ms}$, tone duration $500 \mathrm{~ms}$, pitch difference [0 48263034 ] semi-tones, tone sequence repeated twice. The posterior mode (the sequence combination with the highest posterior probability) was 111111 , i.e. all tones assigned to the same stream. b) Fast sequence, ISI $100 \mathrm{~ms}$, tone duration $100 \mathrm{~ms}$ (posterior mode 121212). e-h) Example of a galloping stream, from [1], e) ISI 26.6ms, pitch difference 6 semi-tones (posterior mode 111) g) ISI 26.6ms, pitch difference 6 semi-tones (posterior mode 121). $\mathrm{c}-\mathrm{d}$ ) and g-h) show the modelled sum posterior distance of the streams. Parameters for this figure (and subsequent figures) were $\alpha=1.44, \sigma=40$.

\section{Context}

An aspect of auditory perception that especially received attention from the Gestalt movement, was the role of context in auditory clustering. Experiments done by [26] showed that modifying the context in which tones were presented modified the segregation of unmodified tones. Figure 3 shows an example, based on ([26]), where two low tones 

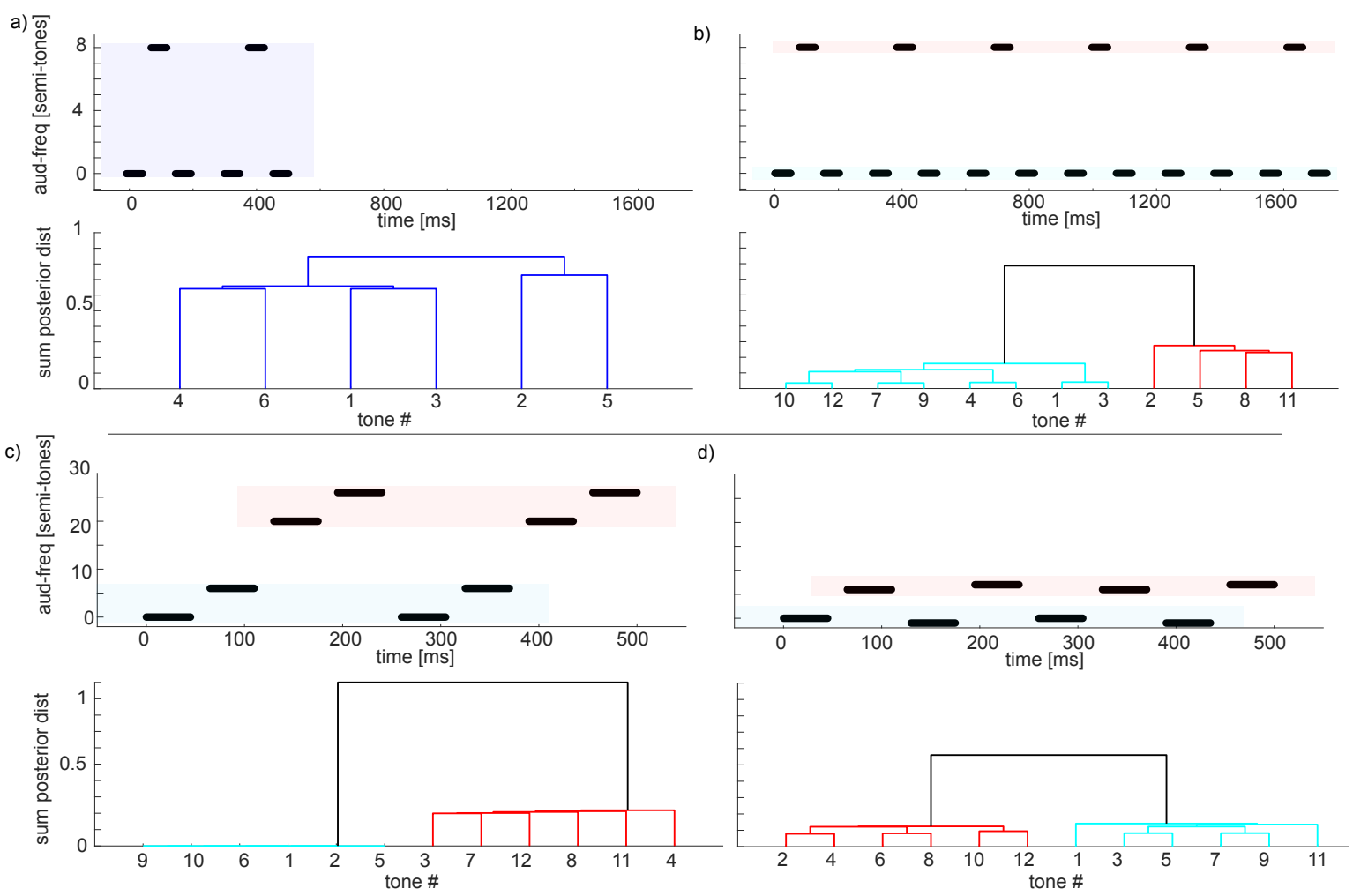

Figure 3. a-b) Stimuli used in experiments from Bregman [26], highlighting the cumulative effect of tones. Stimuli are shown at the top, bottom is dendrogram tree-plots based on the posterior distribution over clustering. A unique colour is assigned to clusters with more than 50 percent distance from other clusters. a) Short sequence ISI $26.6 \mathrm{~ms}$, pitch difference 7 semi-tones, tone sequence repeated twice (posterior mode 111). b) Long sequence ISI $26.6 \mathrm{~ms}$, pitch difference 7 semi-tones, tone sequence repeated eight times (posterior mode 121). c-d) Context matters for the clustering of tones. Stimuli are shown at the top, at the bottom are dendrogram tree-plots based on the posterior distribution over clustering. A unique colour is assigned to clusters with more than 50 percent distance from other clusters. c) Two low tones, two high tones, leading to low tones segregated from high tones (posterior mode 1122) d) While the two low tones have been kept constant, the context of the two other tones now causes them to be clustered separately with the other tones (posterior mode 1212). Long sequence ISI $26.6 \mathrm{~ms}$, tone sequence repeated eight times. Modeling parameters were the same as in Fig 2

will be clustered together while two distractor tones are far off in frequency, but will be clustered separately as the distractor tones are placed around them. The model replicates this phenomena, showing a separation of the first two tones in Fig 3c-d. 


\section{Crossing}

As shown by Tougas and Bregman ([27]) interleaving a decreasing and increasing series of tones gives the illusory percept of the two streams 'bouncing' i.e. the lower set of tones are clustered and segregated from the higher set of tones. Figure 4 recreates this experiment with $2 \times 10$ interleaved decreasing and increasing tones. The model recreates the perceptual phenomenon, with the lower frequency tones grouped together, separate from the higher frequency tones, thus implying a perceived 'bounce'.
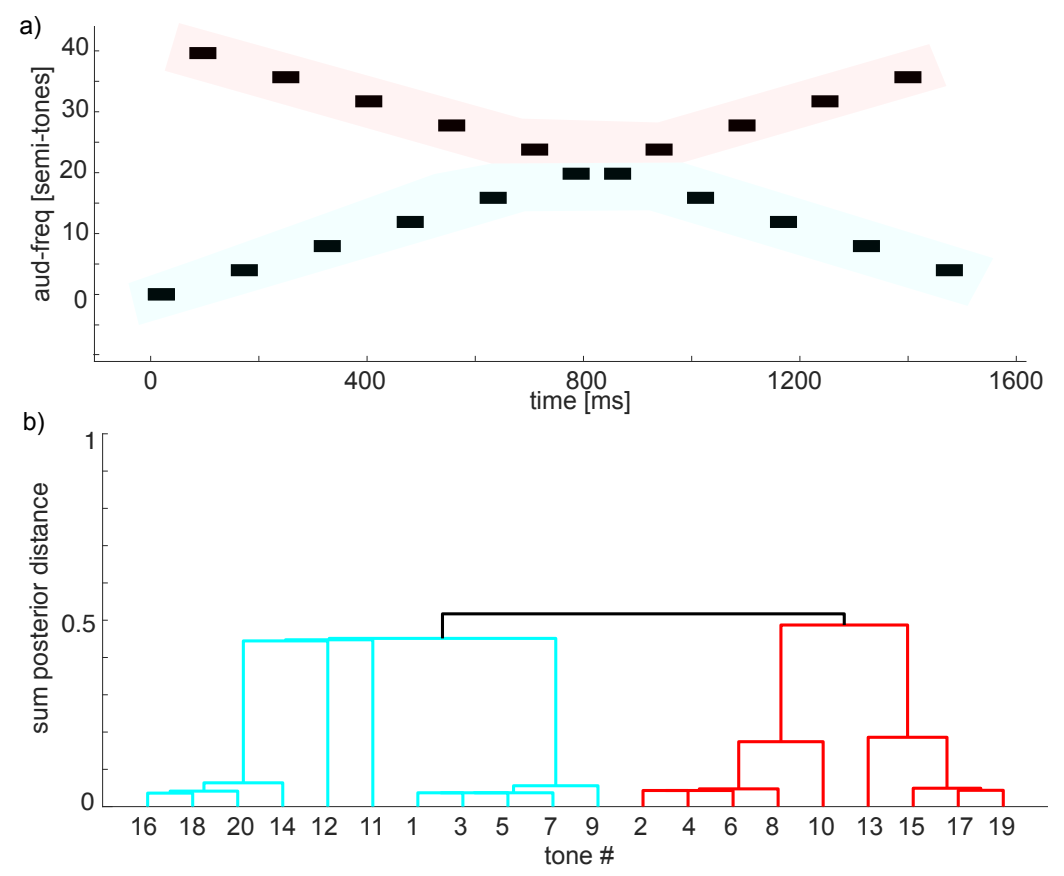

Figure 4. Interleaved increasing (unven numbered tones) and decreasing (even numbered tones) series of tones, ISI $26.6 \mathrm{~ms}$. Same as for human observer the model assigns higher value to a

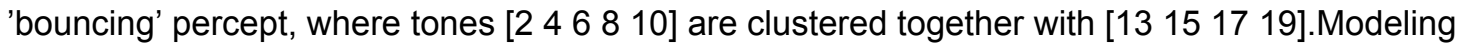
parameters were the same as in Fig 2 
Overall, the model is able to recreate several phenomena in the experimental literature.

\section{Experiment 1}

To quantitatively compare the model to human performance, we conducted a psychophysics experiment, in which fifteen participants with normal hearing listened to simple auditory sequences and performed a subjective judgement task (a variant of the galloping experiment [1], see Figure 2). Given a sequence of Low-High-Low tones, subjects would respond whether they perceived one or two streams. Across trials, the separation between low and high tones, and the inter-stimulus-interval, were varied (see Methods for details).

\section{Model performance and comparison}

As an example, response data from six subjects is shown in Figure 5b. As expected, when the ISI was short, or when the difference in frequency was large, subjects were more likely to report two streams than one. Figure 5 also shows the model fit for one participant.

While visually the model approximates the subject behaviour, we used model-comparison to rule out other hypotheses.

The model with the non-parametric prior was compared against three alternatives that used different priors to constrain the number of possible streams:

A. When the stream combination comprised only one stream (repeated), the prior probability of the next stream being 1 or 2 was allocated according to the CRP, but if the combination already contained two streams, the prior probability of allocating stream 1 or 2 was simply the fraction of previous tones that were allocated to stream 1 or 2 respectively.

B. The prior probabilities of a new tone being allocated to stream 1 or stream 2 were given by P1 and 1-P1, respectively, where $\mathrm{P} 1$ is a fixed parameter.

C. The prior probabilities of a new tone being allocated to stream 1 or 2 were fixed at 0.5 .

Because the alternative model $\mathrm{C}$ has only one free parameter (all others have two), we use the Bayesian information criterion $(B I C=-2 \log P($ resp $\mid$ tones $)+k * \log (n)$, where $k$ is the number of parameters and $\mathrm{n}$ is the number of data points fitted over) to compare model performance in Figure 5. The Bayesian model with the non-parametric process prior gives a better fit (smaller BIC) than all the alternatives considered. The mean \pm SEM of the optimised parameters for the unconstrained model are $\alpha=3.01 \pm 0.25$ (equivalently $P(11)=0.273 \pm 0.030$ ) and $\sigma=123.8$ \pm 6.2 [semitones/sec]. 
a) Model, subject 8

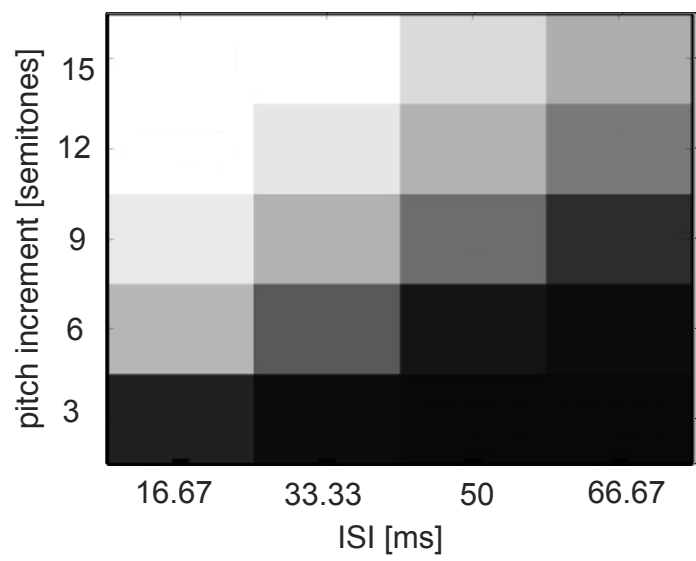

b)

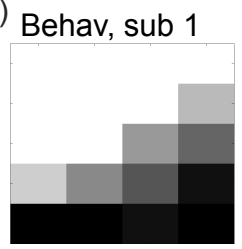

Behav, sub 10

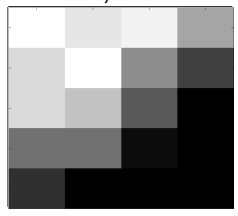

Behav, sub 8

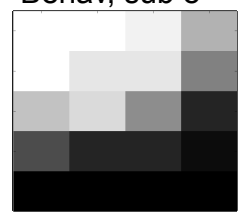

Behav, sub 5

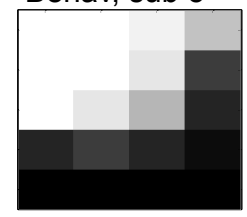

Behav, sub 3

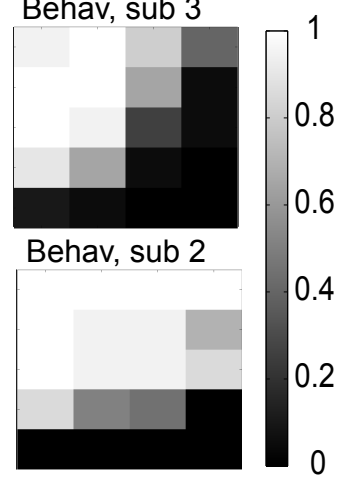

c)

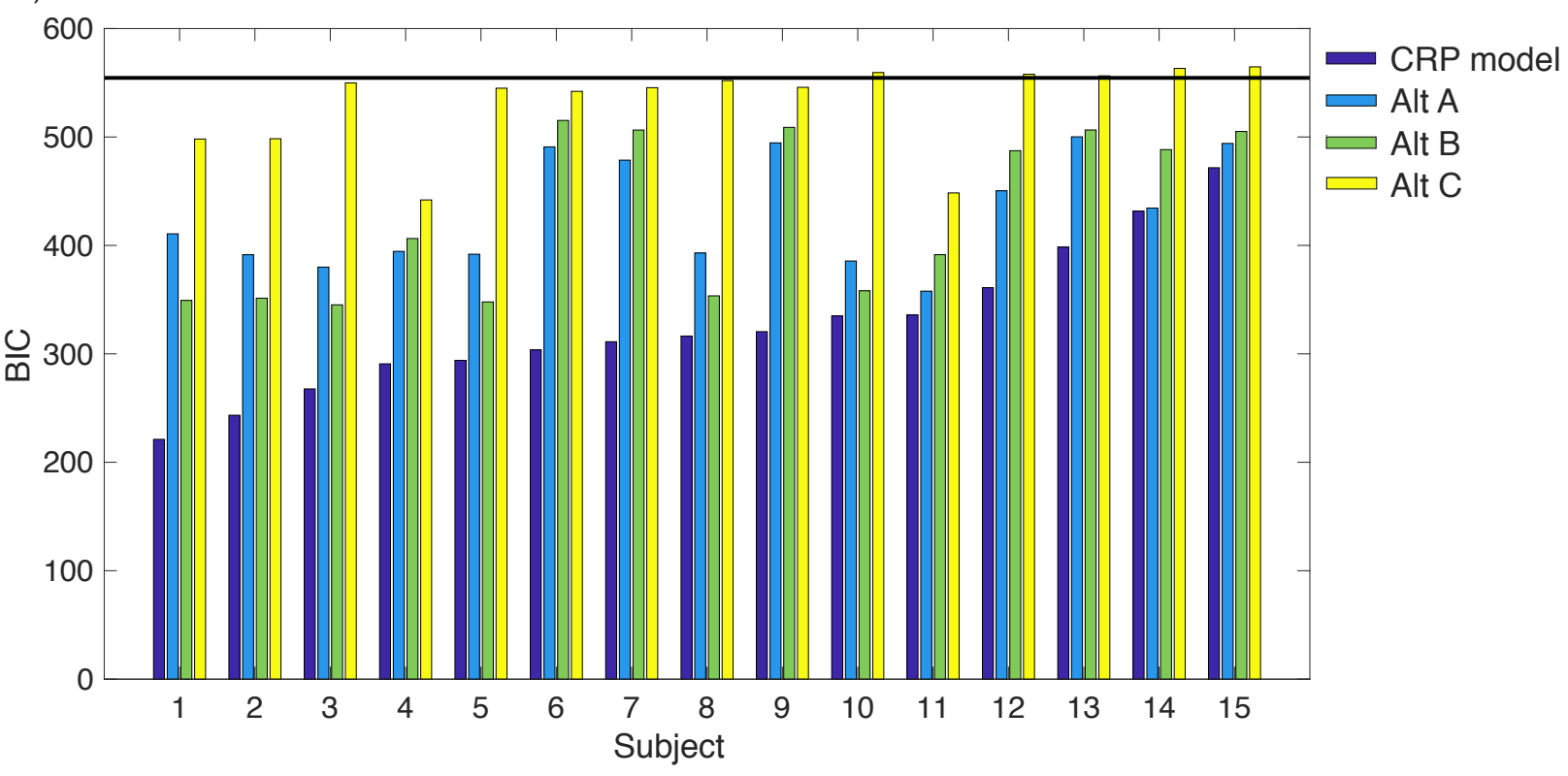

Figure 5. a) Model fit, based on fitted parameters from subject KC, giving the fraction of trials in which the participant responded ' 2 ' for the number of streams perceived. Axes give the pitch difference for the middle tone and the inter stimulus interval (ISI): the time between the offset of one tone and the onset of the next. b) The behavioural results from 6 subjects. c) Model performance on experiment 1 in terms of Bayes Information Criterion (BIC) for each subject with the CRP model (dark blue), alternative A (light blue), alternative B (green), and alternative C (yellow). The black line indicates the performance of a purely random model that assigns 0.5 probability to either response for every condition. Subjects are ordered based on CRP model BIC values. 


\section{Experiment 2}

While the model above theoretically allows an unlimited number of tones to be segregated into an unrestricted number of streams, Experiment 1 (presented above) only allows a repeated sequence of 3 tones to be separated into 1 or 2 streams. However, the model predicts that subjects should generally segregate based on the frequency and temporal distance between tones with a possibly infinite number of streams. To test this further, we performed a novel follow-up experiment in a broader auditory environment. The experimental setting was inspired by Barsz [28] and was specifically designed to allow for a 3-streams situation to emerge, and to replace the explicit measure of stream segregation by an implicit one (see Methods for details).

Participants were asked to judge if two consecutive melodies comprised of 4 repeated tones were similar or different. In every condition, 1 tone was considered being in the low frequency range, 2 tones in the medium range, and 1 tone in the high range. According to previous experiments showing that order information is intact when tones are inside the same stream but lost when segregated into different streams ([11, 28, 29]), if medium tones were to be part of a stream excluding low and high tones, participants should be unable to detect the difference between two sequences if only medium tones were inverted. Thus, subjects should only be able to detect the inversion of the middle tones if the tones are placed in the same stream as the upper or lower stream (hence all tones clustered into 1 or 2 groups). Conditions were created with varying discrepancies between frequency ranges (ISIs were kept constant for this experiment). See Figure 7 for a schematic representation of a typical stimulus with inversion.

As our model predicts that the probability of being assigned to different streams is dependent on the frequency difference, our first prediction is that participants should have a reasonable degree of performance in detecting a difference between two melodies when the medium tones are inverted and there is only a small frequency difference between those medium tones and at least one of the high and low tones. This would reflect the fact that medium tones are in a stream also including other tones. Conversely, our other prediction is that as the frequency difference increases, participants should perform significantly worse at detecting the medium tone inversion. This would reflect the fact that medium tones are in a stream not including other tones.

\section{Analysis preparation}

Individual responses for perceived differences between sequences were transformed into D-prime scores to obtain a single measure of signal detection for each pair of frequency differences, while taking into account possible response biases. Two participants with a negative D-prime score on the easiest condition (3-3) were considered unable to perform the task correctly and were therefore excluded from further analysis, leaving a total of 24 participants. No data was missing in the dataset. A one-way repeated measures ANOVA was conducted on these D-prime scores, with frequency differences (3-3 vs. $3-9$ vs. $3-15$ vs. 9-9 vs. 9-15 vs. 15-15) as the only within-subject factor, along with seven subsequent paired-sample t-tests in line with our hypotheses. 


\section{Data analysis}

Although inferential statistical tests were conducted on D-prime scores only, table 1 also includes the summary statistics of the proportions of "similar" responses in every experimental condition.

One-way repeated measures ANOVA (frequency differences) showed that D-prime scores differed as a function of this factor $[F(5,115)=6.669, p<.001]$.

Seven subsequent paired-samples t-tests were conducted to decompose the main effect of frequency differences over D-prime scores. Three one-tailed paired-samples t-tests revealed that D-prime scores were significantly higher in the 3-3 condition than in the 9-9 condition $\left[t(23)=3.003, p=.003, d_{z}=0.613\right]$, in the $9-15$ condition $[t(23)=2.556$, $\left.p=.009, d_{z}=0.522\right]$ and in the $15-15$ condition $\left[t(23)=2.535, p=0.009, d_{z}=0.517\right]$. Two two-tailed paired-samples ttests revealed no significant difference between the $3-3$ and the $3-9$ conditions $\left[t(23)=0.681, p=.503, d_{z}=0.139\right]$ and between the $3-3$ and the $3-15$ conditions $\left[t(23)=-1.568, p=.131, d_{z}=-0.32\right]$. Another two two-tailed paired-samples t-tests revealed no significant difference between the $9-9$ and the $9-15$ conditions $\left[t(23)=-1.02, p=.318, d_{z}=-0.208\right]$ and between the 9-9 and the 15-15 conditions $\left[t(23)=-0.949, p=.353, d_{z}=-0.194\right]$. These results are summarized in Figure 6.

\begin{tabular}{|c|c|c|c|c|c|c|}
\hline & $3-3$ & $3-9$ & $3-15$ & $9-9$ & $9-15$ & $15-15$ \\
\hline $\begin{array}{c}\text { Different } \\
\text { stimuli } \\
\text { pre- } \\
\text { sented }\end{array}$ & 0.2708 & 0.2882 & 0.2396 & 0.4861 & 0.4965 & 0.5382 \\
\hline $\begin{array}{c}\text { Similar } \\
\text { stimuli } \\
\text { pre- } \\
\text { sented }\end{array}$ & 0.7986 & 0.7708 & 0.8333 & 0.7708 & 0.8542 & 0.8958 \\
\hline \hline
\end{tabular}

Table 1. Mean proportions of "similar" responses and mean D-prime scores across all conditions. Reported errors are \pm 1 standard deviation 


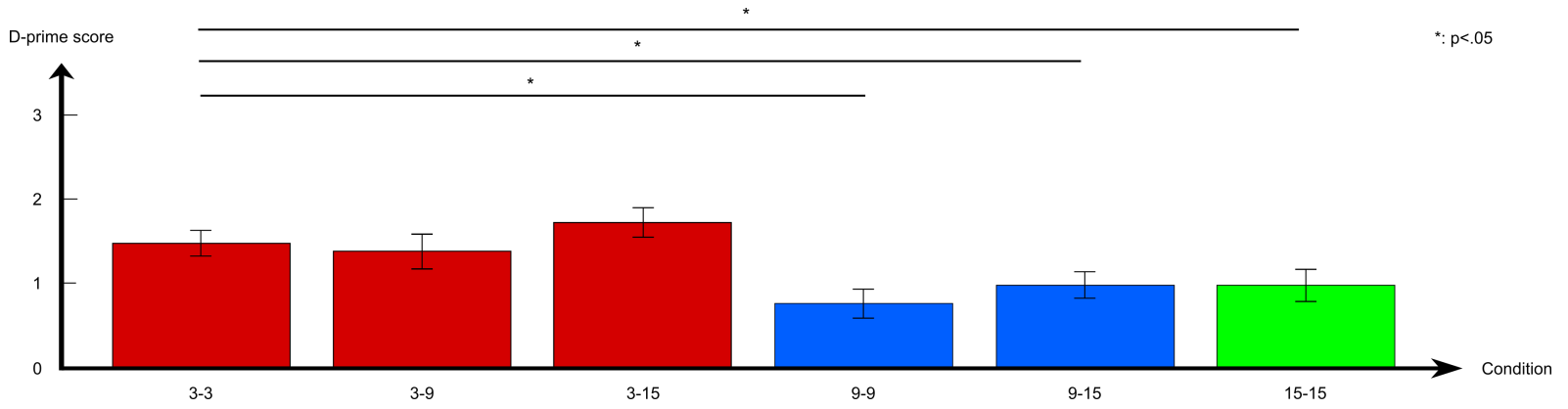

Figure 6. D-prime scores as a function of frequency difference. Red bars indicate conditions with a small minimum frequency difference, blue bars indicate conditions with an intermediate minimum frequency difference and green bars indicate conditions with a large minimum frequency difference. Error bars are \pm 1 standard error

\section{Discussion}

We have presented a simple Bayesian perceptual model that is able to assign stimuli to an unrestricted number of sources, through clustering of stimuli. We have applied the model to the specific case of auditory stream segregation, an area where Gestalt psychology has long emphasized the need for grouping. Utilizing a non-parametric Bayesian prior the model iteratively updates the posterior distribution over the assigned group of each stimulus and provides an excellent description of the perceptual interpretation of simple auditory sequences by human observers.

With just two parameters, the model gives a good account of the basic characteristics of auditory stream segregation - the variation in the probability of perceiving a single sound source as a function of the repetition rate and pitch difference of the sounds. The basic model (with a softmax decision function) gave a better fit to experimental data (Exp. 1) than alternative models that were constrained to interpret the sounds as being produced from just one or two streams. Predictions from the model were also in accordance with results from a novel experiment with a larger number of tones (Exp. 2).

Importantly, the model goes beyond giving just the number of sources, but says which sounds are produced by each source. While the combinatorial space of the posterior distribution in experiment 1 was collapsed to give a marginal distribution in a continuous 1-d response space (leading to an estimate of response probability), the maximum a posterior (MAP) for all participants was always located at either $111-111 \ldots$ or $121-121 \ldots$, depending on the stimulus condition (figure 2). This is reassuring as it is consistent with the anecdotal evidence that participants always perceive either a galloping rhythm (streams $111-111 \ldots$ ) or a high-pitch and a low pitch stream (121-121...), i.e. the percept is always at the MAP. Indeed, the percept cannot in general be at the mean because the space of possible percepts is discrete: there is no percept in between 111 and 121 . 
One consequence of the inference model that is not addressed by mechanistic models of stream segregation is that when a percept changes from, say 111-111 to $121-121$, the source allocation of previous sounds is changed. Ironically, this 'non-causal' effect is essentially a feature of causal inference - when an observer decides that the percept has changed to 121-121, this is based on previous evidence, and yet at the time that the previous tones were heard, they were all associated with one source. A similar effect is commonly encountered when mis-interpreted speech (perhaps mis-heard due to background noise) suddenly makes sense when an essential word is heard - the previous words are reinterpreted, similar to the letters in predictive text message systems.

In the current implementation we had to make numerical approximations in order to handle the complexity of the model. As an alternative to calculating our results analytically, but with approximations) we could use Monte Carlo techniques (e.g. Markov Chain Monte Carlo sampling, i.e. MCMC, a different type of approximation), which have become a standard tool for solving complex statistical models. While not presented here, we have also implemented a MCMC version of the model with similar results. In future work this might be useful at explaining bistable behavioural phenomena where an observer will seemingly randomly switch between incongruous interpretations of stimuli [30, 31].

The framework of the model is very general and allows for the incorporation of other factors into the likelihood to describe other aspects of auditory stream segregation. Adding terms in the likelihood function may be able to explain other effects seen in the literature, such as segregation based on bandwidth ([32]), or build-up and resetting of segregation ([33]). Furthermore, in the current study we assume that there is no ambiguity in the percept of the pure tones, the uncertainty arises from the lack of knowledge about the underlying generative structure of the data. In a realistic situation perceptual ambiguity would have to be taken into account using an approach such as suggested by Turner and Sahani ([34]). Nevertheless, we should emphasize that even though we are dealing with a Markov property (each tone within a stream only depends on the previous tone within the stream), the mixture of streams makes the problem very different from the work on e.g. Hidden Markov Models (or even Infinite Hidden Markov Models) for which the goal is to infer the underlying states despite noisy signal [35].

Our approach is an example of a predictive coding model [36] that uses a generative model to make predictions about relationships between stimuli, and subsequently update such relationships. The model shares similarities with work by Barniv and Neike [30], which used Gaussian Mixture models to describe the integration and segregation of auditory objects, and assumed that a new object could be introduced with a small fixed probability. The model implemented by Chambers et al. [37] also used a similar probabilistic framework and Gaussian likelihood to explain the perceived continuity of pure tones with octave relationships ('Shepard tones'). In contrast to such probabilistic models there have also been proposals for mechansitic models, such as neural network models simulating the abstract processing of the cochlea and auditory cortex [38], as well as models explaining the behavioural phenomena through interactions between different neural populations [15].

The proposed model of auditory stream segregation is a specific instantiation of an iterative probabilistic approach 
towards inference of perceptual information. A major issue for this approach is the problem of dealing with multiple sources, as represented by the work done on causal inference ([2, 3]). Until now, models of causal inference have been unable to handle more than two sources, due to the escalating number of parameters needed for parametric priors. The use of a non-parametric prior allows a complex of many stimuli to be interpreted without running into this problem, potentially allowing for an arbitrary number of causes in the world. This approach is very general - it can be applied to any set of discrete sequential cues involving multiple sources - and it gives a simple, principled way to incorporate natural signal constraints into the generative model.

While frequency and time are the most studied dimensions in the Auditory Scene Analysis literature $[9,12]$, the general idea of explaining perceptual clustering through the use of a Bayesian non-parametric prior can potentially be applied to other auditory variables, such as harmonic complex tones [39] or multiple speakers, i.e. the cocktail party problem [40]. It is also possible to add other features in addition to time and frequency, to the exising model, such as spatial discrepancy and saliency, topics we plan to explore in the future. ${ }^{* * *}$ The auditory streaming model currently also does not consider attentional mechanisms. As here presented it assumes that our perceptual system automatically groups or segregates cues into a potentially infinite number of streams based on stimulus characteristics, and that attention only has the role of selecting one or several clusters of interest in a given situation, or is being driven by the posterior probability of the different clusters. In either case, attentional processes would be entirely independent of the mechanisms simulated by the model. Nevertheless, it has already been proposed that attention could interact with the cluster formation process itself and therefore affect their overall configuration [41]. If that is the case, the model's responses could significantly differ from behavioural results in situations where top-down attention is being manipulated.

Auditory stream segregation is considered a typical example of the Gestalt principle of Pragnanz [4], according to which properties of a set of stimuli are interpreted in a manner that simplifies the interpretation. We would propose that another way to interpret this is by the principle of clustering, with stimuli that are similar (in auditory frequency, visual continuity etc.) being combined into a single simple interpretation. The simplification principle is naturally interpreted in our framework through a non-parametric Bayesian process that naturally restricts the complexity of the interpretation, such as the number of possible objects or sources in a scene.

In conclusion, we have shown that auditory scene perception of streams of single frequency tones can be explained by a simple Bayesian model utilizing a non-parametric prior. This highlights the importance of clustering in auditory perception, although the approach is applicable to any combination of stimuli and perceptual cues. Together with advances in visual perception [7] this hints at clustering being a general property of perception. 


\section{Methods}

\section{Modelling}

\section{Model response}

To determine the response of the model to a tone sequence, the posterior for each possible sequence, $S_{1: n}$, is calculated tone-by-tone until all 30 tones (maximum) have been presented. To relate the final posterior over sequences to response $r_{k}$ of subject $k$, ('1 or 2 streams') $P_{\text {model }}\left(r_{k} \mid\right.$ tones), we assume that subjects maximise the expected utility

$$
\underset{r_{k}}{\operatorname{argmax}}<U>=\underset{r_{k}}{\operatorname{argmax}} \sum_{i, j} U\left(r_{k}, S_{i}, S_{j}\right) P\left(S_{i}=S_{j} \mid f, t\right)
$$

where the utility of a response given two tones in same stream is counted as 1 if they are in the same stream, and zero otherwise. Note that the absolute values of $S_{i}$ do not matter, just whether they are in the same stream or not.

$$
\begin{array}{ll}
U\left(r_{k}, S_{i}, S_{j}\right)=1 & \text { if }\left(r_{k}=1, S_{i}=S_{j}\right) \text { or }\left(r_{k}=0, S_{i} \neq S_{j}\right) \\
U\left(r_{k}, S_{i}, S_{j}\right)=0 & \text { if }\left(r_{k}=0, S_{i}=S_{j}\right) \text { or }\left(r_{k}=1, S_{i} \neq S_{j}\right)
\end{array}
$$

The best response is then to choose 1 (single stream) if

$$
\sum_{i, j} P\left(S_{i}=S_{j} \mid f, t\right)>\sum_{i, j}\left(1-P\left(S_{i}=S_{j} \mid f, t\right)\right)
$$

If the observer believes all tones came from the same stream they should choose $r_{k}=1$, if they are convinced half the tones are from one stream, half from another they should choose $r_{k}=2$.

We assume soft-max, a variant of probability matching similar to Luce's law ([42]), to explain the variability in data and allow us to fit our models.

$$
P\left(r_{k} \mid f, t\right)=\frac{\exp \left(\beta * \sum P\left(S_{i}=S_{j} \mid f, t\right)\right)}{\exp \left(\beta * \sum P\left(S_{i}=S_{j} \mid f, t\right)\right)+\exp \left(\beta * \sum\left(1-P\left(S_{i}=S_{j} \mid f, t\right)\right)\right)}
$$

The parameters of the model (as well as for the alternative models) were optimised using the BADS toolbox (as a more robust alternative to MATLAB's fminsearch routine, [43]) to maximise the log-likelihood of the data, log $P_{\text {model }}\left(r_{k} \mid\right.$ tones, par $)$ independently for each subject $k$. During each iteration of the search, a sequence of 30 tones was presented to the model for each condition, and the probability of response ' 1 ' was calculated per condition. 


\section{Model posterior approximation}

Using the iterative scheme above, we can calculate analytically the possible combinations of tones, but as the tone sequence progresses the number of possible source combinations - and hence the size of the posterior distribution increases exponentially. To prevent combinatorial explosion two methods were used to generate an approximation of the full posterior distribution. The first limits the number of tones that are retained when using the previous posterior as the next prior, i.e. the algorithm only retains the last 30 tones and their potential allocations to sources

Limiting the number of tones eases the computational load and can also be seen as a crude model of a limited memory capacity. Although the iteratively constructed prior retains some stream information of all previous tones, when a very short memory is used, this may not be sufficient to generate stable stream allocation as the CRP prior probabilities fluctuate greatly when the number of previous tones is small. Furthermore, if the structure of the sequence is an important cue for streaming, a larger memory may be necessary to determine regularities in the sequence.

Even when the memory is limited to (e.g.) the previous six tones, allocating a stream to the seventh tone requires a posterior distribution taking 858 values, most of which must necessarily have very small probabilities. A second method to limit the size of the posterior is simply to select only the most probable stream combinations by imposing a probability threshold, hence we only propagated stream combinations with $P\left(S_{1: n} \mid x_{1: n}\right)>0.001$. Together, these approximation methods allow a reasonable memory length of 30 tones (to avoid instability), while avoiding combinatorial explosion.

\section{Experimental setup}

Two experiments were conducted to test different aspects of the model. Experiment 1 was a replica of the 'galloping' stimuli experiment ([1]) performed to test the quantitative performance of the model, while the second experiment was a novel task designed to test one of the predictions of the model.

Subjects for both experiments were under-graduate students and received course credits for their participation, except for one of the authors who participated in Experiment 1. Each subject was fully briefed, provided informed consent and was given brief training on the task they performed. Experiments were completed by different subjects. No personal data was kept, ensuring participants' anonymity. The two experiments were approved by University of Birmingham and Durham University's Ethics Committees respectively.

Stimuli were dynamically programmed using Matlab (Mathworks, 2018) on a PC desktop computer. Both experiments used the Psychtoolbox extension to ensure timings were accurate [44]. Stimuli were played through Sennheiser 280 headphones at a comfortable supra-threshold level plugged into an external sound card. The experiment was carried out in a special sound-attenuated room. 


\section{Experiment 1}

This experiment replicated the study from [1], using fifteen participants. Figure 2 shows a schematic of the stimuli used - each sequence comprised 30 tones in repeated LHL- triplets, where the dash represents a silent gap. Each tone was $50 \mathrm{~ms}$ in duration, including $10 \mathrm{~ms}$ raised cosine onset and offset ramps. A $4 \times 5$ factorial design was used: the pitch of the high tones took values of $3,6,9,12$, and 15 semitones above the low tone, which had a fixed frequency of $1000 \mathrm{~Hz}$, and the offset to onset interval took values $17,33,50$, and $67 \mathrm{~ms}$. The duration of the silent gap was equal to the tone duration plus the offset-onset interval. Conditions were ordered randomly - each condition was tested 20 times over 5 runs, each run lasting approximately 7 minutes. At the end of the sequence participants pressed a key to report whether the percept at the end of the sequence was most like a single stream (a galloping rhythm) or two separate streams of notes. The whole experiment lasted about 50 minutes.

\section{Experiment 2}

Twenty-six participants were enrolled for this study. No personal data was kept, ensuring participants' anonymity.

Material and stimuli Each testing trial consisted in 2 sequences of 4 pure tones in repeated Low-Medium-HighMedium (L-M1-H-M2 or L-M2-H-M1) quadruplets. The first sequence was always repeated 22 times for a total of 88 tones presented. The second one was always repeated a total of 11 times for a total of 44 tones presented. Tones between sequences within a same trial were always the same and only their order of presentation could differ. Each tone was $100 \mathrm{~ms}$ in duration, including $10 \mathrm{~ms}$ raised cosine onset and offset ramps. The offset to onset interval between tones inside a sequence was $16.67 \mathrm{~ms}$. Each sequence also had general $500 \mathrm{~ms}$ long raised cosine onset and offset ramps. The offset to onset interval between sequences inside a trial was $2 \mathrm{~s}$. The lowest tone had a fixed frequency of $440 \mathrm{~Hz}$ across trials. The highest possible tone had a frequency of $2960 \mathrm{~Hz}$. The lowest frequency was specifically chosen to correspond to a common tone, and to control for differences in perceived loudness as much as possible across the range of played frequencies. Indeed, the $440-2960 \mathrm{~Hz}$ range presents a low variability in equal-loudness [45] The frequency of M1 was calculated in semitone increases from the lowest tone, according to experimental conditions. M2 was always 3 semitones higher than M1. As was the case for the difference between $\mathrm{L}$ and $\mathrm{M} 1$, the frequency of $\mathrm{H}$ was calculated in semitone increases from M2, in relation to experimental conditions (see figure 7 for a representation of a typical trial).

Training trials were similarly designed and consisted in 2 sequences of 3 pure tones in repeated Low-MediumMedium (L-M1-M2 or L-M2-M1) triplets.

Design A 2x6 within-subjects factorial design was used. The first independent variable was the medium tones inversion, which could be either present or absent between the 2 sequences of a trial. This resulted respectively 


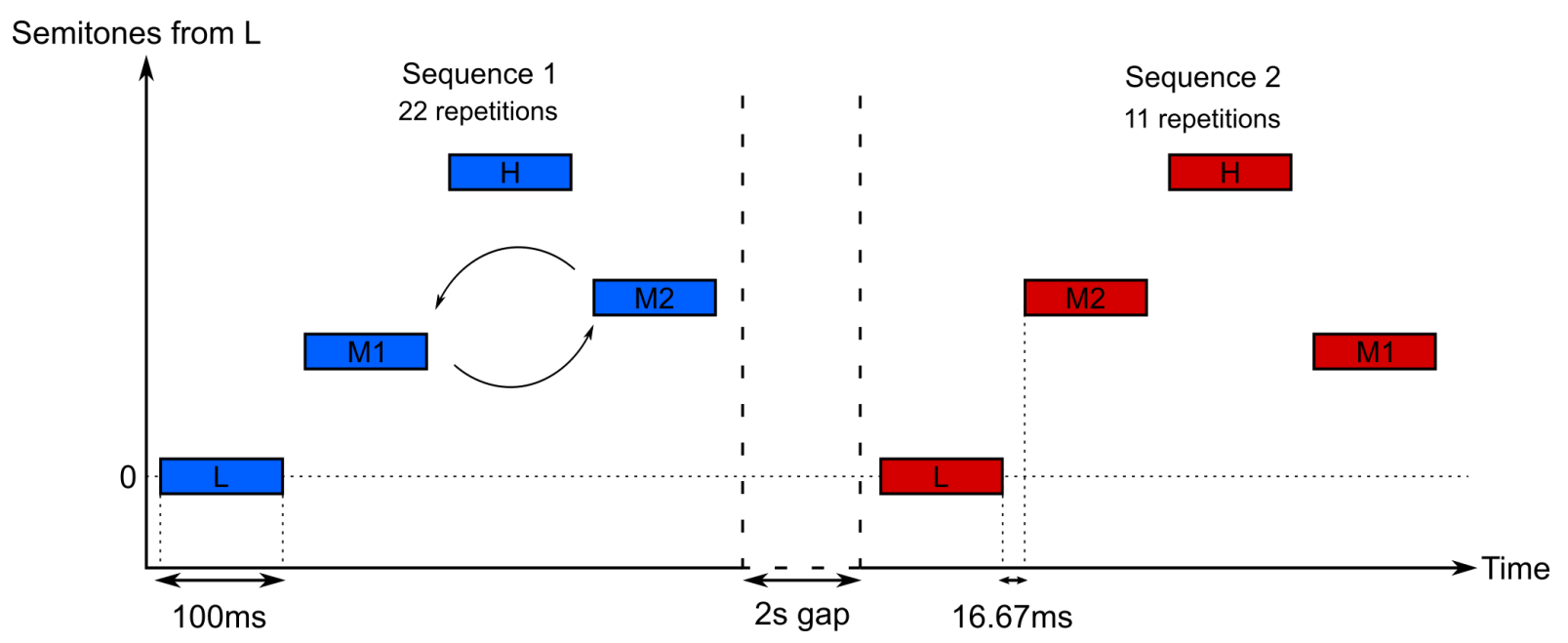

Figure 7. Visual representation of a trial with inversion in a 9-9 frequency difference condition

in trials objectively comprised of a pair of different sequences, and trials objectively comprised of a pair of twin sequences. The second independent variable was pairs of frequency differences between $L$ and $M 1$, and between $M 2$ and $\mathrm{H}$, counted in semitones. Possible values were 3-3, 3-9, 3-15, 9-9, 9-15, and 15-15. Conditions were presented randomly, and the order in which baseline and inverted tone sequences were presented was counterbalanced. Each identical pair of sequences was presented 6 times, while each different pair of sequences was presented 12 times for a total of 108 trials. Training trials consisted in 3 identical pairs and 3 different pairs repeated twice, for a total of 12 training trials.

The first dependent variable was the perceived difference between sequences (different vs. similar). The second dependent variable was the level of confidence in this judgement (on a scale from 1 to 4 , 4 being "very confident"). We did not analyse the confidence judgments here.

Procedure Participants were greeted in a small sound-attenuated room and were asked to sit at a desk, approximately $60 \mathrm{~cm}$ from a computer screen. They were handed an information and a privacy notice sheet, stating the general aims of the experiment, their rights as a subject, and how their data would be handled. After reading and asking any questions they may have to the experimenter, they were asked to sign a consent form. They were then asked to put the headphones on once they confirmed they understood the task instructions.

Participants were asked to listen to pairs of melodies presented sequentially, and judge after each pair if the melodies were similar or different by pressing the right key on a keypad ("1" for different, "2" for similar). They were also asked to rate their confidence about the judgement they just made, by pressing a key from 1 to 4 on the same keypad. Participants were warned that tones between sequences had the same frequency, and that they should focus on the order of tones within the melody. On each trial, a white dot was displayed in the middle of the screen for a brief 
period to signify a new trial was about to start. A grey dot was then displayed in place of the white one along with the instruction "listen" while melodies were being played. A black dot, along with a reminder of the response keys, replaced them as soon as the melodies were finished, meaning that subjects could enter their response. Participants had no time limit to respond, as the next trial would only start after they did.

Once the experiment was over, the experimenter gave an oral feedback explaining the aims, design, and experimental background of the study. The whole experiment lasted about 55 minutes.

\section{Acknowledgements}

We are very grateful for discussions and helpful comments by Uta Noppeney as well as colleagues at Durham University Psychology department. NL was funded through a Durham University Post-graduate grant.

\section{References}

1. Van Noorden, L. Temporal Coherence in the Perception of Tone Sequences PhD Thesis (University of Technology, Eindhoven, The Netherlands., 1975).

2. Körding, K. et al. Causal inference in multisensory perception. Plos One 2, e943. issn: 19326203 (2007).

3. Shams, L. \& Beierholm, U. R. Causal Inference in Perception. Trends in Cognitive Sciences 14, 1-8. issn: 13646613 (Aug. 2010).

4. Wagemans, J. et al. A century of Gestalt psychology in visual perception: II Conceptual and theoretical foundations. Psychological Bulletin 138, 1218-1252 (2012).

5. Aldous, D. J. Exchangeability and Related Topics. Lecture notes in mathematics 1117, 1-198. issn: 0075-8434 (1985).

6. Ghahramani, Z. Bayesian non-parametrics and the probabilistic approach to modelling. Phil. Trans. R. Soc. A 371, 20110553. issn: 1364503X (2013).

7. Froyen, V., Feldman, J. \& Singh, M. Bayesian Hierarchical Grouping: Perceptual Grouping as Mixture Estimation. Psychological Review 122, 575-597. issn: 0033295X (2015).

8. Gershman, S. J. \& Niv, Y. Perceptual estimation obeys Occam's razor. Frontiers in psychology 4, 623. issn: 1664-1078 (Jan. 2013). 
9. Moore, B. C. J. \& Gockel, H. E. Properties of Auditory Stream Formation. Philosophical transactions of the Royal Society of London. Series B, Biological sciences 367, 919-31. issn: 1471-2970 (Apr. 2012).

10. Anstis, S. M. \& Saida, S. Adaptation to Auditory Streaming of Frequency-Modulated Tones. Journal of Experimental Psychology: Human Perception and Performance, 11, 257-271 (1985).

11. Bregman, A. S. \& Campbell, J. Primary Auditory Stream Segregation and Perception of Order in Rapid Sequences of Tones. Journal of experimental psychology 89, 244-9. issn: 0022-1015 (Aug. 1971).

12. Bregman, A. S. Auditory Scene Analysis the Perceptual Organization of Sound isbn: 978-0-26252195-6 (MIT Press, 1994).

13. Beauvois, M. W. \& Meddis, R. Time Decay of Auditory Stream Biasing. Perception \& psychophysics 59, 81-86. issn: 0031-5117 (1997).

14. Wang, D. Primitive Auditory Segregation Based on Oscillatory Correlation. Cognitive Science 20, 409-456. issn: 03640213 (Sept. 1996).

15. McCabe, S. L. \& Denham, M. J. A Model of Auditory Streaming. The Journal of the Acoustical Society of America 101, 1611. issn: 00014966 (Mar. 1997).

16. Moore, B. \& Gockel, H. Factors Influencing Sequential Stream Segregation. Acta Acustica 88, 320333 (2002).

17. Barlow, H. Possible Principles Underlying the Transformation of Sensory Messages. Sensory Communication, 217-234 (1961).

18. McDermott, J. H. \& Simoncelli, E. P. Sound Texture Perception via Statistics of the Auditory Periphery: Evidence from Sound Synthesis. Neuron 71, 926-40. issn: 1097-4199 (Sept. 2011).

19. Shams, L., Ma, W. J. \& Beierholm, U. Sound-Induced Flash Illusion as an Optimal Percept. Neuroreport 16, 1923-7. issn: 0959-4965 (Nov. 2005).

20. Weiss, Y., Simoncelli, E. P. \& Adelson, E. H. Motion Illusions as Optimal Percepts. Nat Neurosci 5, 598-604 (2002).

21. Beierholm, U. R. in Encyclopedia of Computational Neuroscience 1-5 (Springer Verlag, 2015). doi:10.1007/978-1-4614-7320-6_451-1.

22. Lee, M. D. \& Habibi, A. A Cyclic Sequential Sampling Model of Bistable Auditory Perception in Proceedings of the 31st Annual Conference of the Cognitive Science Society (eds Taatgen, N., van Rijn, H., Nerbonne, J. \& Shonmaker, L.) (2009), 2669-2674. 
23. Turner, R. E. Statistical Models for Natural Sounds PhD Thesis (University College London, 2010).

24. Orbanz, P. \& Teh, Y. W. Bayesian Nonparametric Models 1. 1-14 (Springer, 2010).

25. Wood, F., Goldwater, S. \& Black, M. J. A Non-Parametric Bayesian Approach to Spike Sorting. Conference proceedings : ... Annual International Conference of the IEEE Engineering in Medicine and Biology Society. IEEE Engineering in Medicine and Biology Society. Conference 1, 1165-8. issn: 1557-170X (Jan. 2006).

26. Bregman, A. S. Auditory Streaming Is Cumulative. Journal of Experimental Psychology: Human Perception and Performance 4, 380-387. issn: 1939-1277(Electronic),0096-1523(Print) (1978).

27. Tougas, Y. \& Bregman, A. S. Crossing of Auditory Streams. Journal of Experimental Psychology: Human Perception and Performance 11, 788-798. issn: 00961523 (1985).

28. Barsz, K. Auditory Pattern Perception: The Effect of Tonal Frequency Range on the Perception of Temporal Order. Perception \& Psychophysics 43, 293-303. issn: 0031-5117, 1532-5962 (May 1988).

29. Demany, L. Auditory stream segregation in infancy. Infant Behavior and Development 5, 261-276. issn: 0163-6383 (Jan. 1982).

30. Barniv, D. \& Nelken, I. Auditory Streaming as an Online Classification Process with Evidence Accumulation. PLOS ONE 10, e0144788 (2015).

31. Pressnitzer, D. \& Hupé, J.-M. Temporal Dynamics of Auditory and Visual Bistability Reveal Common Principles of Perceptual Organization. en. Current Biology 16, 1351-1357. issn: 0960-9822 (July 2006).

32. Cusack, R. \& Roberts, B. Effects of Differences in Timbre on Sequential Grouping. Perception \& Psychophysics 62, 1112-1120 (2000).

33. Roberts, B., Glasberg, B. R. \& Moore, B. C. J. Effects of the Build-up and Resetting of Auditory Stream Segregation on Temporal Discrimination. Journal of experimental psychology. Human perception and performance 34, 992-1006. issn: 0096-1523 (Aug. 2008).

34. Turner, R. E. \& Sahani, M. Probabilistic Amplitude and Frequency Demodulation in Advances in Neural Information Processing (eds Shawe-Taylor, J., Zemel, R. S., Bartlett, P., Pereira, F. \& Weinberger, K. Q.) (Red Hook, New York, 2011), 981-989.

35. Fox, E. B., Sudderth, E. B., Jordan, M. I. \& Willsky, A. S. A sticky HDP-HMM with application to speaker diarization. EN. The Annals of Applied Statistics 5. ISBN: 1932-6157 Publisher: Institute of Mathematical Statistics_eprint: 0905.2592, 1020-1056. issn: 1941-7330 (June 2011). 
36. Denham, S. L. \& Winkler, I. Predictive coding in auditory perception: challenges and unresolved questions. en. European Journal of Neuroscience 51, 1151-1160. issn: 0953-816X, 1460-9568 (Mar. 2020).

37. Chambers, C. et al. Prior context in audition informs binding and shapes simple features. Nature Communications 8, 15027 (2017).

38. Krishnan, L., Elhilali, M. \& Shamma, S. Segregating Complex Sound Sources through Temporal Coherence. en. PLoS Computational Biology 10 (ed Lewicki, M.) e1003985. issn: 1553-7358 (Dec. 2014).

39. Moore, B. C. J. \& Gockel, H. E. Resolvability of components in complex tones and implications for theories of pitch perception. Hearing Research 276, 88-97 (2011).

40. Mcdermott, J. H. The cocktail party problem. Current Biology 19, 1024-1027 (2010).

41. Sussman, E. S. Auditory Scene Analysis: An Attention Perspective. Journal of speech, language, and hearing research: JSLHR 60, 2989-3000. issn: 1558-9102 (Oct. 17, 2017).

42. Luce, R. D. On the possible psychophysical laws. Psychological Review 66, 81-95. issn: 0033295X (1959).

43. Acerbi, L. \& Ma, W. J. Practical Bayesian Optimization for Model Fitting with Bayesian Adaptive Direct Search. Advances in Neural Information and Processing Systems 30, 1836-1846 (2017).

44. Kleiner, M. et al. What's new in psychtoolbox-3. Perception 36, 1-16. issn: 0301-0066 (2007).

45. ISO Central Secretary. Acoustics - Normal Equal-Loudness-Level Contours Standard ISO 226:2003 (International Organization for Standardization, Geneva, CH, 2003). https://www.iso.org/standard/ 34222.html. 